\title{
Transiliac sacroplasty for Denis 3 fracture - Two cases report -
}

Received December 22, 2017

Revised 1st, May 22, 2018

2nd, June 10, 2018

Accepted June 18, 2018

\section{Corresponding author}

Wonsok Chang, M.D.

Department of Anesthesiology

and Pain Medicine, Wooridul

Spine Hospital, 445 Hakdong-ro,

Gangnam-gu, Seoul 06068, Korea

Tel: 82-2-513-8485

Fax: 82-2-513-8488

E-mail: ancws@naver.com

ORCID

http://orcid.org/0000-0001-6170-8529

\section{Wonsok Chang', Sangho Lee ${ }^{2}$, and Hojin Kim²}

Departments of ${ }^{1}$ Anesthesiology and Pain Medicine, ${ }^{2}$ Neurosurgery, Wooridul Spine

Hospital, Seoul, Korea

We present two cases of percutaneous sacroplasty for sacral body fracture (Denis 3) and sacral alar fracture under multislice computed tomography (MSCT) guidance and discuss the clinical results and technical considerations. Sacroplasty is often recommended for the treatment of painful sacral insufficiency fractures, which destabilize the sacrum. However, sacroplasty for Denis zone 3 is rare because of the lack of validating controlled studies or unique technical considerations related to sacral anatomy. We performed sacroplasty for Denis zone 3 via the transiliac approach. Precise needle placement and polymethylmethacrylate cement injection were performed safely under the MSCT system. No complications occurred related to this procedure, such as iliac fracture, vascular leakage, or epidural leakage. MSCT-guided transiliac sacroplasty was a useful and effective solution in treating sacral body fracture.

Key Words: Buttock pain, Multislice computed tomography, Sacral body fracture, Sacroplasty, Transiliac approach.
Sacral insufficiency fracture (SIF) is a relatively common cause of debilitating back pain in the elderly. SIFs are estimated to occur in nearly $2 \%$ of women older than 55 years [1]. SIFs are often caused by postmenopausal osteoporosis commonly followed by corticosteroid therapy, rheumatoid arthritis, and pelvic irradiation [2]. Patients with SIFs most commonly present with diffuse lower back pain, which may radiate to the buttock, hip, or groin. Patients may have some tenderness to palpation in the lower back and sacral regions. The diagnosis is often missed on the initial visit, and patients do not receive optimal treatment.

Sacral fractures are classified into zones in accordance with the Denis classification as follows: transalar (zone 1), transforaminal (zone 2), and central (zone 3), with zone 1 fractures being the most common, followed by zone 2 and 3 fractures [3]. Zone 1 corresponds to the ala and is the location of most insufficiency fractures. Zone 1 fractures rarely involve the foramina as in the setting of zone 2 fractures. Zone 3 fractures are located in the central sacral body and are frequently accompanied by neurological deficits and saddle anesthesia.

The traditional therapy for stable SIFs has been absolute bed rest with analgesics followed by physical therapy and osteoporosis medication. However, absolute bed rest can be related to significant morbidity, especially in elderly patients [4]. The most serious problems of absolute bed rest are deep vein thrombus and pulmonary embolism. Their incidence rates have been reported to range from $29 \%$ to $61 \%$ and from $2 \%$ to $12 \%$, respectively [5]. Pneumonia, decubitus ulceration, cardiac dysfunction, bone demineralization, and weight loss can result from absolute bed rest. Recovery may be prolonged, and some patients may develop chronic pain and nonunion requiring surgery.

Sacroplasty has emerged as a minimally invasive alternative to conservative therapy for SIFs [6] and is often recommended for the treatment of painful SIFs that destabilize the sacrum. Brook et al. [7] hypothesized that cements provide

This is an Open Access article distributed under the terms of the Creative Commons Attribution Non-Commercial License (http://creativecommons.org/licenses/by-nc/4.0) which permits unrestricted non-commercial use, distribution, and reproduction in any medium, provided the original work is properly cited. 
fracture stabilization, thus decreasing painful micromotions.

The target of sacroplasty is to provide early symptomatic relief, allowing more rapid mobilization, limiting the need for significant narcotic analgesics. Owing to the lack of validating controlled studies or because of the unique technical difficulty related to the sacral anatomy, sacroplasty for Denis zone 3 fractures is rare. Thus, we adopted the transiliac approach and multislice computed tomography (MSCT) guidance to overcome the technical difficulty. MSCT allows the simultaneous production of three sequential transverse sections and gives more three-dimensional information than conventional computed tomography (CT).

The objective of our study was to determine the possibility and feasibility of MSCT-guided sacroplasty (40-slice Somatom Sensation Open, Siemens, Germany) for Denis zone 3 fractures via the transiliac approach.

\section{CASE REPORTS}

In two patients with Denis zone 3 sacral fractures, transiliac sacroplasty under MSCT guidance was performed. The conservative treatment that was initially performed over a period of 3 weeks was not effective for reduction of the severe, disabling pain.

\section{Case 1}

A 66-year-old woman with osteoporosis presented with 2 months of severe pain in the lower back, pelvic region, and buttock. The T score of bone mineral density was -3.5 . The pain was exacerbated by sitting or standing, and was relieved by assuming the supine position.

Before the sacroplasty, the pain on walking was rated as $8-9 / 10$ on the visual analog scale (VAS) of 0 to 10 . Magnetic resonance imaging (MRI) revealed fractures of the S1 sacral body and both alae (Fig. 1A).

The patient had no specific significant trauma history. SIFs are stress fractures that result from normal stress on abnormally weak bones and are most frequently observed in the setting of osteoporosis.

The absolute bed rest and analgesic medication over the 3 -week period did not achieve a satisfactory reduction of the severe disabling pain. We discussed with the patient the potential risks and benefits of transiliac sacroplasty and those of conservative medical management. After serious discussion with the patient and her family regarding the procedure, the patient decided to undergo transiliac sacroplasty.

The procedure was performed under conscious sedation with midazolam and fentanyl, and continuous monitoring. A single-shot antibiotic (cefazolin $1 \mathrm{~g}$ intravenously) was routinely administered immediately before the intervention to provide coverage against skin flora. The patient was placed in the prone position under the MSCT scanner. MSCT in an axial slice orientation with multiplanar reformations was then performed before the operation.

Preoperative MSCT (axial spiral with a layer thickness of 5 $\mathrm{mm}$ and coronal and sagittal reformations with a reconstruction interval of $2 \mathrm{~mm}$ ) of the pelvis was performed, and the entry point was marked on the skin. The entry point was established by tilting the MSCT scan gantry to find a safe pathway that does not cross the anatomical landmarks such as the neural foramen and cortical margins of the sacrum. The

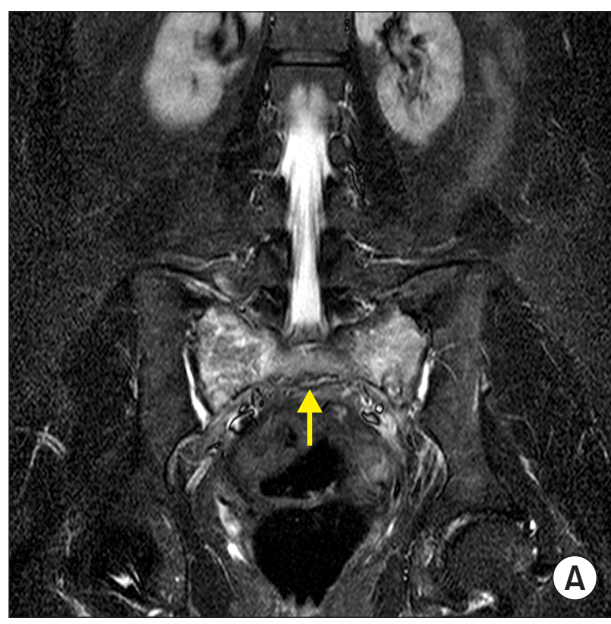

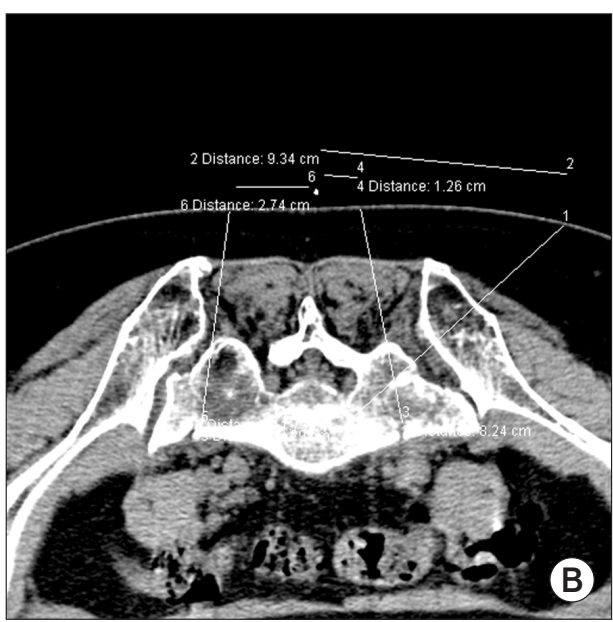

Fig. 1. (A) Fractures of the S1 sacral body (white arrow) and both alae. (B) The skin entry points for the alar fractures are identified using a MSCT scanner. MSCT: multislice computed tomography. 
skin entry points for the alar fractures were also checked (Fig. 1B). After establishment of the skin entry point by using a CT scan, the usual preparation was performed and the respective approach angle was checked. Skin anesthesia was induced, and the muscle and periosteum were anesthetized using $1 \%$ lidocaine.

Under MSCT guidance using the thin-slice technique, an 11-G percutaneous vertebroplasty (PVP) needle (Hyun Medical, Korea) was introduced to the iliac bone via the transiliac approach from lateral to medial via the trajectory previously determined on the CT scanner [8]. We confirmed the placement of the needle tip within the sacral vertebral body without compromising the sacral canal and sacral neural foramens (Fig. 2). After removing the inner needle, a polymethylmethacrylate (PMMA) mixture (CMW1, DePuy International Ltd., UK) was injected to the sacrum under MSCT
(Fig. 3) by using 1-ml syringes. The cement, which was in a toothpaste consistency, was injected with the needle directed laterally to encourage the flow of the cement away from the sacral foramen. The goal of the PMMA injection was to fill the fracture site and surrounding bone without extravasation of the cement. Cement filling was performed discontinuously under MSCT guidance. PMMA cement was injected in 0.5-ml aliquots, and the total volume of cement injected to the sacral body fracture site was $2.5 \mathrm{ml}$. The sacral alar fractures were treated after PMMA injection to the S1 sacral body.

An 11-G PVP needle was then advanced into the respective fracture zone in the sacrum from a dorsal to a ventral direction (short axis), and cement filling was performed discontinuously $(0.5 \mathrm{ml})$ under MSCT guidance. Furthermore, 4.1 (left) and $3.7 \mathrm{ml}$ (right) of cement was injected via each cannula (Fig. 4A). The patient was then allowed to walk 2 hours after
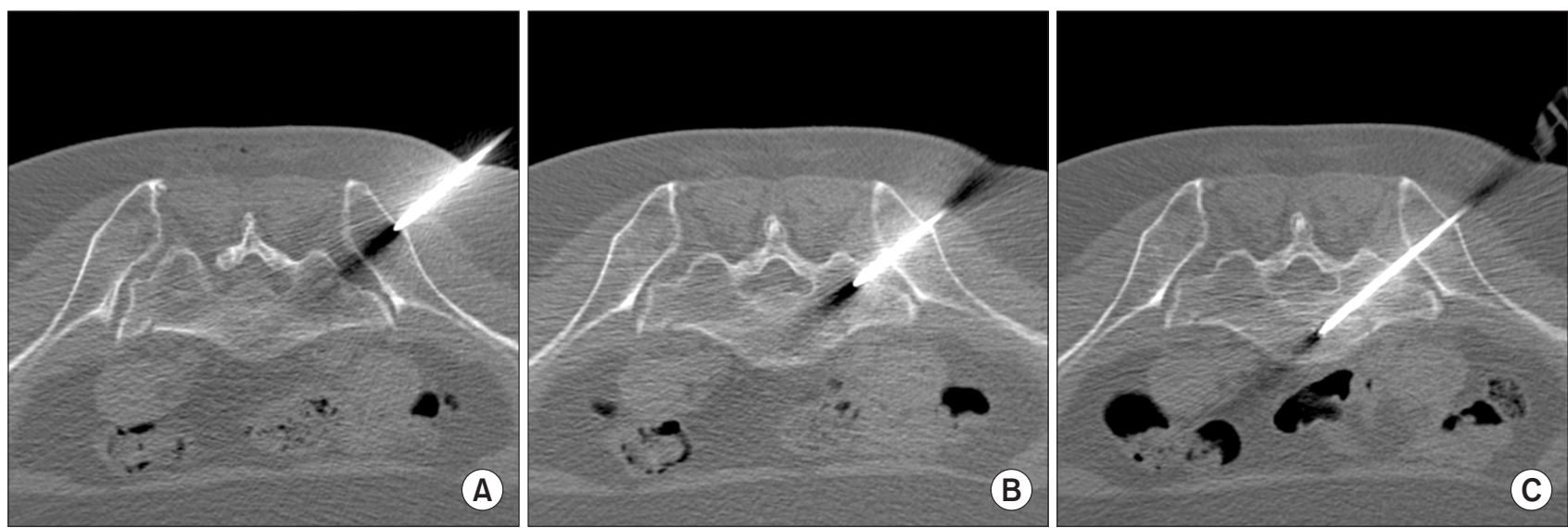

Fig. 2. A PVP needle is introduced via the transiliac approach under MSCT guidance. PVP: percutaneous vertebroplasty, MSCT: multislice computed tomography.
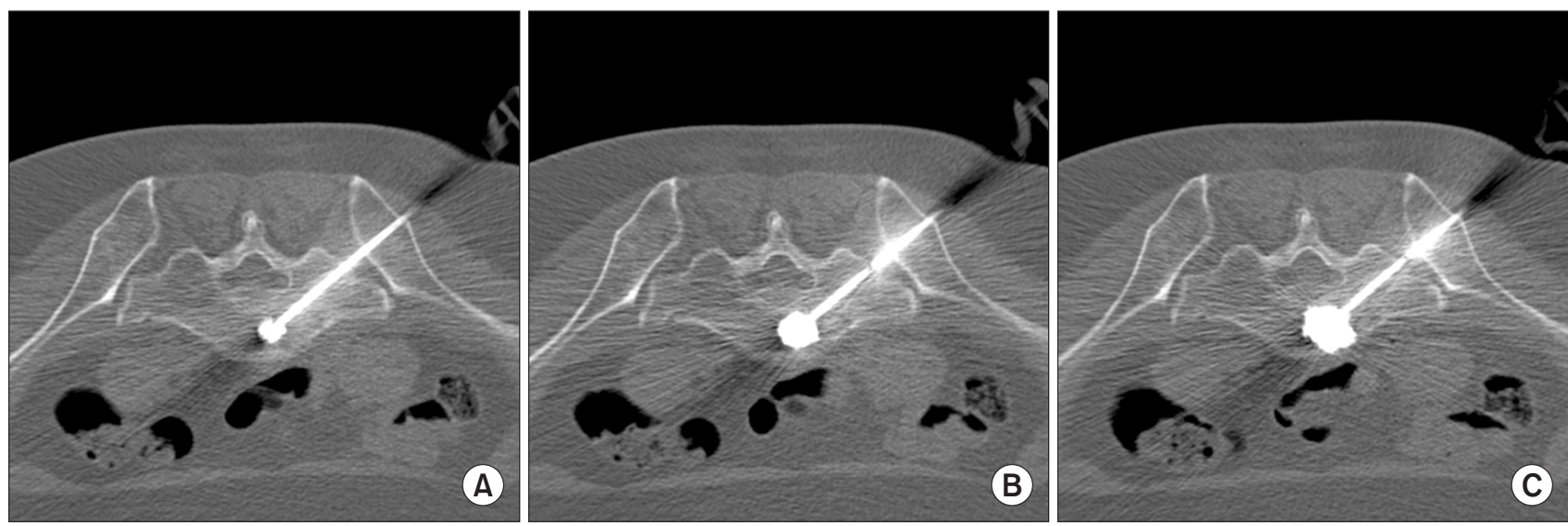

Fig. 3. Bone cement is injected to the sacral body under serial MSCT monitoring. MSCT: multislice computed tomography. 


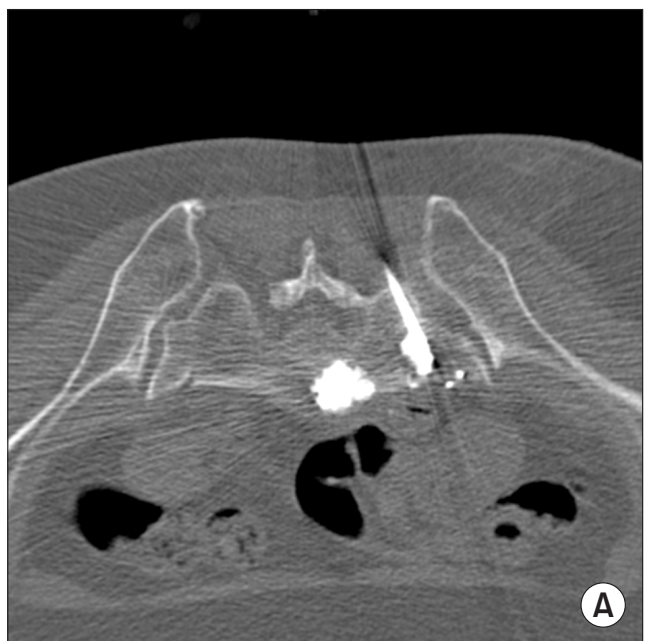

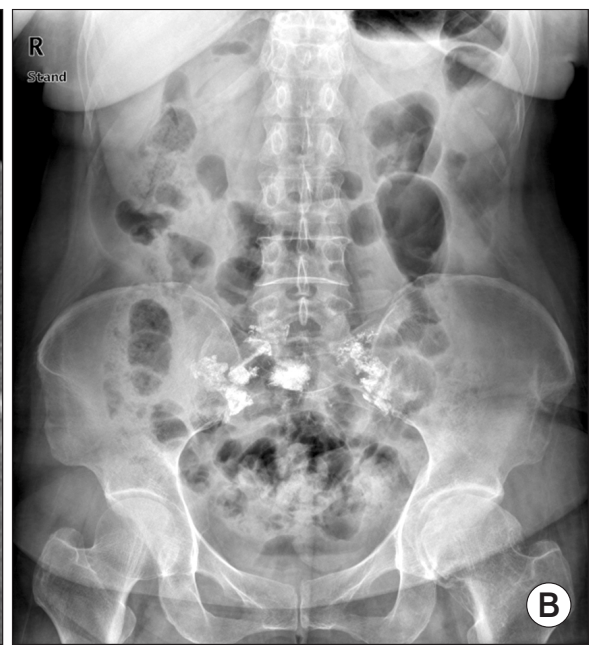

Fig. 4. (A) Bone cement is injected via each cannula. (B) A radiograph showing good distribution of cement over the area of the sacral fracture.
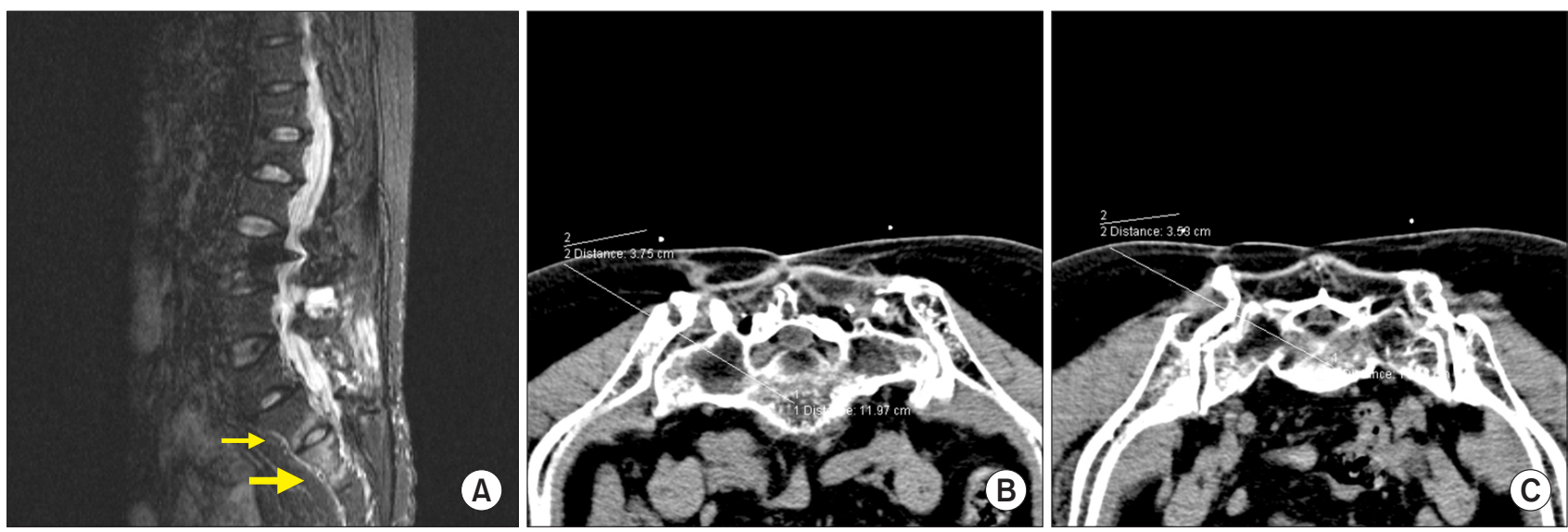

Fig. 5. (A) An MRI scan demonstrating sacral $\mathrm{S} 1$ and $\mathrm{S} 2$ body fractures (white arrows). (B, C) The entry point is established by tilting the MSCT scan gantry to find a safe pathway that does not cross the anatomical landmarks. MRI: magnetic resonance imaging, MSCT: multislice computed tomography.

the procedure.

The pain intensity in the buttock area was determined using the VAS before the intervention, 2 days after the intervention, and 1 month after the intervention. The VAS pain score was 8 before the intervention, and a significant reduction in pain to a VAS score of 3 was observed on the second postoperative day, which stabilized after 1 month to a VAS score of 4 . No complication occurred related to the procedure, such as iliac fracture, at the 1-month follow-up. Transiliac sacroplasty was technically feasible under the MSCT-guided technique. The MSCT scan showed a central distribution of the cement in the sacrum and a good distribution over the area of the sacral fracture (Fig. 4).

\section{Case 2}

A 73-year-old woman with a history of osteoporosis was admitted after a slip injury. Fifteen years ago, she underwent lumbar posterior fusion at $\mathrm{L} 3 / \mathrm{L} 4$ with pedicle screw fixation and PVP at L1 at another hospital. She reported severe coccygeal and buttock pains limiting her motions. A subjective pain assessment revealed an 8/10 pain score, which was not controlled by analgesic drug administration and injection therapy. The pain had been preventing her from sitting and walking normally. An MRI scan demonstrated sacral S1 and S2 body fractures without an alar fracture (Fig. 5A). We performed MSCT-guided percutaneous sacroplasty at the S1 and S2 sacral bodies via the transiliac approach by using the same method. A preoperative MSCT scan of the pelvis was performed, and the skin entry points at S1 and S2 were 
marked. The entry point was established by tilting the MSCT scan gantry to find a safe pathway that does not cross the anatomical landmarks such as the neural foramen and cortical margins of the sacrum (Figs. 5B and 5C). The amount of cement required depended on the extent of the fracture and PMMA leakage. Furthermore, $2.0 \mathrm{ml}$ of PMMA cement was introduced at the S1 sacral body (Fig. 6) and $1.6 \mathrm{ml}$ at the S2 sacral body (Figs. 7A and 7B). The post-procedural CT and radiography revealed the optimal filling of the lesions with no evidence of PMMA extravasation into the central canal or sacral foramina (Fig. 7C). The VAS pain score significantly decreased from 9 preoperatively to 3 on the second postoperative day and 3 in the first month after the intervention, and the fracture of the patient was successfully mobilized.

\section{DISCUSSION}

The first percutaneous sacroplasty for the treatment of an insufficiency fracture was described by Garant [6] in 2002. The complicated anatomy of the sacrum should be taken into account in the technical performance of sacroplasty because of the oblique configuration of the sacrum and its foramina. The technical challenges are inherent in the safe placement of needles and prevention of cement extravasation. For this reason, a CT-guided procedure is helpful for the placement of PMMA near the adjacent vascular, neurological, and visceral structures [9].

Pommersheim et al. [10] suggested that CT fluoroscopy provides both the precision of CT and the direct visualization of cement delivery through real-time fluoroscopy. They suggested that CT alone is insufficient for sacroplasty because the real-time monitoring offered by fluoroscopy is necessary for early detection of cement migration. However, the timing and accuracy of such a procedure were vital for the PMMA injection because of the short period to PMMA solidification. CT and fluoroscopy do not provide views of the operation
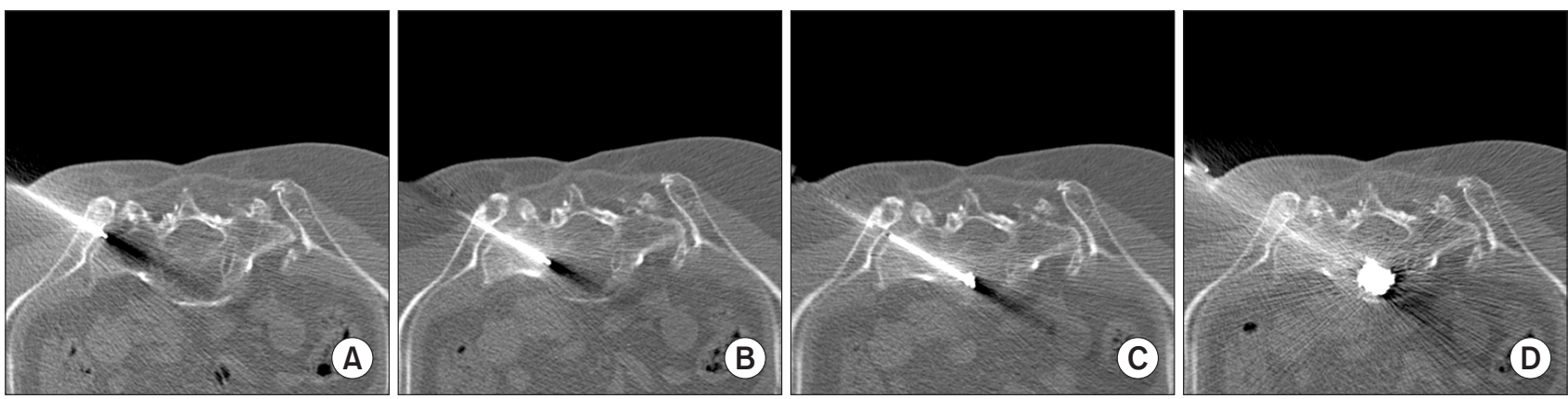

Fig. 6. PMMA cement is introduced at the $\mathrm{S} 1$ sacral body. PMMA: polymethylmethacrylate.
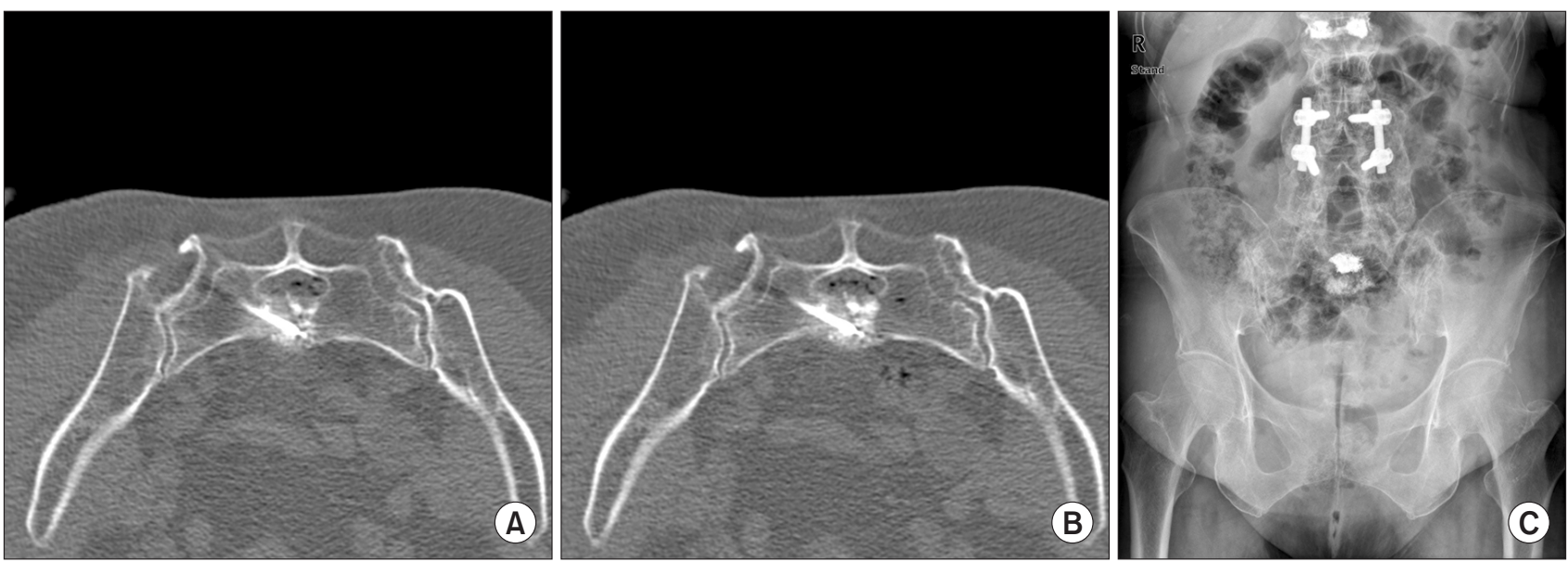

Fig. 7. (A, B) PMMA cement is introduced at the S2 sacral body. (C) A radiograph showing optimal filling of PMMA at the S1 and S2 sacral bodies. PMMA: polymethylmethacrylate. 
field at the same time. Thus, either CT or fluoroscopy should be selected during the procedure. Real-time fluoroscopy is a good modality for vertebroplasty. However, the complexity of the sacral anatomy limits the application of fluoroscopy for detecting extrusion of cements into the sacral foramina and epidural space. With fluoroscopy, it can be difficult to determine whether the needle has adequately traversed the outer cortex and has not breached the inner cortex on the pelvic side [11]. If the injection volume is introduced slowly, threedimensional CT could allow an accurate needle placement and monitor the PMMA injection during the procedure. As MSCT can show the upper and lower levels simultaneously, it can provide a better definition of spatial relationships within the treatment field, so that injuries near vascular, neurological, and visceral structures could be prevented. We injected PMMA mixtures in $0.5 \mathrm{ml}$-aliquots and performed each injection under MSCT.

The role of the sacral bodies in inducing the pain related to SIFs is not clearly understood. Although several studies $[1,9,12]$ suggest that pain relief from sacroplasty is typically good, many physicians who routinely treat SIFs often observe that the pain relief from sacroplasty is not as complete as the relief that can be achieved with the treatment of the thoracic or lumbar vertebral segments [12]. Although not specifically studied, it could be the reason for the sacral body being a source of sustained pain despite good stabilization of the lateral sacral elements [13].

Owing to the presence of the iliac crest and posterior iliac spine, treatment of $\mathrm{S} 1$ vertebral body fractures has remained a challenge for physicians, even with CT imaging. We attempted to overcome this obstacle by using the transiliac approach. The transiliac approach in percutaneous endoscopic lumbar discectomy was already used in patients without complications [14]. With the MSCT-guided transiliac approach, the needle could be more easily advanced to the sacral body without making contact with the sacral nerve and spinal canal.

In this case report, transiliac sacroplasty relieved disabling pain rapidly, which resulted in increased mobility and improved quality of life. This procedure was safe and practicable for reducing intractable pain when performed with precise MSCT guidance and a thorough appreciation of the complex sacral anatomy. Therefore, in conclusion, transiliac sacroplasty can be a useful therapeutic option in the overall pallia- tive treatment of patients with sacral body fractures (Denis zone 3 fractures).

\section{REFERENCES}

1. Weber M, Hasler P, Gerber H. Insufficiency fractures of the sacrum. Twenty cases and review of the literature. Spine (Phila Pa 1976) 1993; 18: 2507-12.

2. Grasland A, Pouchot J, Mathieu A, Paycha F, Vinceneux P. Sacral insufficiency fractures: an easily overlooked cause of back pain in elderly women. Arch Intern Med 1996; 156: 668-74.

3. Denis F, Davis S, Comfort T. Sacral fractures: an important problem. Retrospective analysis of 236 cases. Clin Orthop Relat Res 1988; 227: 67-81.

4. Babayev M, Lachmann E, Nagler W. The controversy surrounding sacral insufficiency fractures: to ambulate or not to ambulate? Am J Phys Med Rehabil 2000; 79: 404-9.

5. Lin JT, Lane JM. Sacral stress fractures. J Womens Health (Larchmt) 2003; 12: 879-88.

6. Garant M. Sacroplasty: a new treatment for sacral insufficiency fracture. J Vasc Interv Radiol 2002; 13: 1265-7.

7. Brook AL, Mirsky DM, Bello JA. Computerized tomography guided sacroplasty: a practical treatment for sacral insufficiency fracture: case report. Spine (Phila Pa 1976) 2005; 30: E450-4.

8. Sciubba DM, Wolinsky JP, Than KD, Gokaslan ZL, Witham TF, Murphy KP. CT fluoroscopically guided percutaneous placement of transiliosacral rod for sacral insufficiency fracture: case report and technique. AJNR Am J Neuroradiol 2007; 28: 1451-4.

9. Butler CL, Given CA 2nd, Michel SJ, Tibbs PA. Percutaneous sacroplasty for the treatment of sacral insufficiency fractures. AJR Am J Roentgenol 2005; 184: 1956-9.

10. Pommersheim W, Huang-Hellinger F, Baker M, Morris P. Sacroplasty: a treatment for sacral insufficiency fractures. AJNR Am J Neuroradiol 2003; 24: 1003-7.

11. Deen HG, Nottmeier EW. Balloon kyphoplasty for treatment of sacral insufficiency fractures. Report of three cases. Neurosurg Focus 2005; 18: e7.

12. Whitlow CT, Mussat-Whitlow BJ, Mattern CW, Baker MD, Morris PP. Sacroplasty versus vertebroplasty: comparable clinical outcomes for the treatment of fracture-related pain. AJNR Am J Neuroradiol 2007; 28: 1266-70.

13. Betts A. Vertebroplasty of the first sacral vertebra. Pain Physician 2009; 12: 651-7.

14. Choi G, Kim JS, Lokhande P, Lee SH. Percutaneous endoscopic lumbar discectomy by transiliac approach: a case report. Spine (Phila Pa 1976) 2009; 34: E443-6. 